\title{
O DESAFIO DA FORMAÇÃO EM ENGENHARIA DE PETRÓLEO NO CONTEXTO DA PANDEMIA SARS-COV-2: UMA PROPOSTA PARA REALIZAÇÃO DE EXPERIMENTOS SOBRE TENSÃO SUPERFICIAL
}

DOI: $10.37702 / 2175-957 X . C O B E N G E .2021 .3612$

Julia Silva Cavalcante de Aguiar - juliaaguiar@id.uff.br

Universidade Federal Fluminense

Rua Otávio Carneiro 43

24230-190 - Niterói - RJ

Messias Gutemberg de Moura Vieira Junior - messiasjunior@id.uff.br

Universidade Federal Fluminense

Rua Passo da Pátria 30

24210-240 - Niterói - RJ

Rogério Fernandes de Lacerda - rogeriolacerda@id.uff.br

UFF Universidade Federal Fluminense

Rua Vera Crispino de Freitas 48

24210-327 - Niterói - RJ

Geraldo de Souza Ferreira - geraldoferreira@id.uff.br

Universidade Federal Fluminense

Rua Passo da Pátria 156

24210-240 - Niterói - RJ

Resumo: Considerando as dificuldades impostas pela pandemia do COVID-19, este trabalho busca facilitar a viabilização de um experimento relevante para 0 segmento de óleo e gás, por meio da proposta de um aparato de simples construção. $O$ estudo escolhido foi a medição de tensão superficial e interfacial que tem importância em fenômenos que ocorrem a altas temperaturas e pressões, utilizando o método do peso da gota. A tensão superficial e interfacial é uma grandeza com significativo potencial para fornecer informações relevantes para a solução de alguns problemas presentes na indústria de petróleo. No desenvolvimento é apresentada a relevância, os desafios e a estruturação do experimento. Por fim, é feita uma reflexão sobre a significância deste estudo sob a ótica das partes envolvidas. 


\section{(C) COBENGE

Palavras-chave: Laboratório. Tensão superficial e interfacial. Indústria de petróleo. Experimento. Pandemia sara-covid-2 


\section{O DESAFIO DA FORMAÇÃO EM ENGENHARIA DE PETRÓLEO NO CONTEXTO DA PANDEMIA SARS-COV-2: UMA PROPOSTA PARA REALIZAÇÃO DE EXPERIMENTOS SOBRE TENSÃO SUPERFICIAL}

\section{INTRODUÇÃO}

Desde os primórdios da profissão, pode-se considerar de maneira geral que o papel do engenheiro após sua formação é aliar as competências técnicas, seja de cunho teórico ou prático, às habilidades designadas como soft skills, para aplicar seus conhecimentos na resolução de problemas, geração de novos conhecimentos e concepção de resultados práticos à sociedade.

Tendo em vista essas atribuições, com enfoque nas experiências práticas, tem-se a utilização das aulas em laboratório como a maneira com que as instituições formadoras aproximam os egressos às situações vivenciadas no campo durante o exercício profissional. O protagonismo desse ambiente pode ser ressaltado na afirmação "dentro da concepção de formação do engenheiro para atender as necessidades da sociedade, o laboratório é um elemento de importância estratégica", (CAMPOS, 2012, p. 40).

$\mathrm{O}$ atual período de isolamento social, devido à pandemia vigente, obriga muitos cientistas a pensar em alternativas para atenuar o impacto desse distanciamento nas atividades de pesquisa. A ciência não pode parar. Apesar das restrições impostas, a quarentena não pode significar uma interrupção completa dos trabalhos de produção científica.

Com a atual conjuntura mostra-se necessário ampliar as possibilidades de investigação, experimentando novos modos de fazer pesquisa. A atividade científica não está declinando nesse período, mas passa por mudanças na estruturação de suas prioridades. "Os pesquisadores focam em atividades que não dependem de reuniões presenciais ou da utilização de equipamentos laboratoriais" (ÁLVARES, 2020).

Assim, o momento atual exige mudanças não apenas na forma de pensar e fazer ciência, mas também na relação que estabelecemos com nossos desafios. Em uma realidade onde posicionar-se de maneira mais ativa não é possível, a inovação e a adaptação se mostram fundamentais para continuar a evolução.

$\mathrm{Na}$ presente oportunidade foi projetado um experimento para a medição de tensão superficial e interfacial em laboratório. A estimativa de valores desta grandeza é relevante pois ela toma parte em processos que ocorrem na indústria de petróleo em altas temperaturas e pressões. Neste sentido, conhecer a tensão superficial e interfacial permite que os alunos possam contribuir na elaboração de métodos de viabilização de contribuições para a indústria de petróleo.

\section{METODOLOGIA}

Foi realizada uma pesquisa bibliográfica para obtenção do embasamento conceitual necessário ao conhecimento da importância dos fenômenos de superfície na Engenharia de Petróleo e para obtenção de subsídios teóricos para construção do aparato experimental para mensuração de valores sobre a tensão superficial.

As pesquisas foram feitas em livros clássicos de Engenharia de Petróleo, presentes na biblioteca virtual da Universidade Federal Fluminense (UFF), e artigos científicos da base Science Direct, acessada pelo portal de Periódicos da Capes (Coordenação de Aperfeiçoamento de Pessoal de Nível Superior). Informações sobre os meios e materiais 
necessários (tubos, válvulas, tê, cruzetas, manômetros, termômetros, etc.) para construção do aparato experimental foram obtidas em catálogos de fabricantes e fornecedores da construção civil.

\section{RELEVÂNCIA DO ESTUDO PARA A INDÚSTRIA DE ÓLEO E GÁS}

Desde a fase inicial de construção da indústria de petróleo tal como a conhecemos hoje, partindo da perfuração do primeiro poço de petróleo pelo Cel. Drake em 1859, na Pensilvânia, nos Estados Unidos, essa indústria passou por avanços tecnológicos expressivos. Com uma atividade intensa de exploração em busca do petróleo e seus derivados, em um contexto de demandas crescentes por combustíveis, a indústria de petróleo e gás natural teve que enfrentar o desafio de se mover em direção offshore (em ambiente marinho), alcançando profundidades cada vez maiores com condições cada vez mais complexas e severas (THOMAS, 2012).

Estas dificuldades culminaram em uma evolução técnica muito alta da mão de obra responsável pelas atividades, com suporte em uma filosofia de resolução de problemas e uma fomentação de aprimoramentos contínuos, formando o que se designa hoje como engenheiro de petróleo. No dia a dia da produção de um campo de petróleo, cabe ao engenheiro, no exercício de suas atividades, lidar com uma grande quantidade de tomadas de decisão, tanto no planejamento quanto na execução dos procedimentos corriqueiros. Tendo isto em vista, quanto mais questões puderem ser previstas e evitadas, maior a taxa de sucesso das operações industriais.

A busca constante por novas fontes de energia tem sido uma das grandes preocupações da humanidade. Neste cenário, a pesquisa e a exploração de novas jazidas petrolíferas têm um papel de destaque. Num contexto onde os recursos revelam-se cada vez mais escassos, a economicidade de qualquer empreendimento industrial, seja relativo à exploração de recursos naturais ou não, passa a ser um fator decisivo para a sua viabilidade. Atualmente, os centros de pesquisa têm como um de seus principais objetivos o desenvolvimento e aplicação de técnicas capazes de aumentar a economicidade de um campo de petróleo, quer seja pela antecipação da produção, quer seja pelo aumento do fator de recuperação do campo.

De acordo com Kodera et al. (2020), dentre os vários entraves e desafios enfrentados pela indústria de petróleo na época contemporânea, e para os quais se buscam soluções, podem ser citados:

- O "plugging" - que é a obstrução das tubulações que levam o petróleo do poço até à unidade de produção de petróleo, devido à formação de hidratos de gás;

- A imprecisão na previsão do fator de recuperação de óleo - pois a incerteza acerca da influência das forças capilares nas regiões porosas prejudica a acurácia da simulação do reservatório;

- A problemática do sequestro de $\mathrm{CO} 2$ - que é uma atividade essencial à minimização dos danos ambientais ao lidar com o dióxido de carbono, produzido nos poços em associação com o petróleo;

- A incerteza gerada em torno de vazamentos em grandes profundidades;

- Compreender o comportamento da fase e as propriedades da superfície dos fluidos de reservatórios que exibem comportamento retrógrado.

O que esses problemas têm em comum é o fato de estarem ligados ao estudo das tensões superficiais e interfaciais associadas ao fluxo de fluidos nos reservatórios de petróleo e nas tubulações durante a produção. A tensão superficial é a medida da força de coesão no limite entre duas fases de uma mistura. Quando os líquidos são imiscíveis, em sua interface é caracterizada a tensão interfacial. Estes fenômenos de superfície são 
explicados pelas forças de Van der Waals. As moléculas no interior de um líquido estão, em média, sujeitas às mesmas forças de atração em todas as direções. Já as que se encontram na interface líquido-ar estão submetidas a forças de atração não-balanceadas, que resultam em uma força em direção ao interior do líquido. Assim, as moléculas tendem a se deslocar para o interior do líquido, contraindo-se espontaneamente. Isso explica a forma esférica adquirida por gotículas de líquidos (SHAW, 1970).

Experimentos realizados, tendo como objetivo conhecer os condicionantes das tensões superficiais em diversas situações da indústria de petróleo, têm um enorme potencial para preencher as lacunas de conhecimento e fornecer informações relevantes para a busca de soluções para os desafios pontuados por Kodera et al (2020).

Os saberes sobre os fenômenos de superfície encontram ampla aplicação no estudo das influências de forças capilares em meios porosos nos reservatórios de petróleo. Apesar da importância do conhecimento sobre os fenômenos de superfície, ainda há uma escassez significativa de dados e informações sobre a tensão superficial em condições extremas de temperatura e de pressão.

A motivação para o presente artigo está inserida neste contexto, pretendendo-se contribuir para sistematizar estudos e projetar experimentos para estimar valores de tensões superficiais em condições extremas de pressão e temperatura.

\section{EXPERIMENTO: MEDIÇÃO DE TENSÃO SUPERFICIAL E INTERFACIAL EM ALTAS PRESSÕES E TEMPERATURAS}

\subsection{Contextualização}

A estimativa da tensão superficial sob alta pressão e alta temperatura pode ser difícil por causa das soluções aquosas em análise que se comportam de uma forma não ideal sob tais circunstâncias. Os valores experimentais desses parâmetros, não estão disponíveis na literatura dos estudos cotidianos e devem, portanto, ser determinados por correlações ou por medições experimentais diretas.

O conteúdo científico disponível propõe vários métodos experimentais para a medição de tensão superficial:

1. Capillary rise method;

2. Stallagmometer method - drop weight method;

3. Wilhelmy plate or ring method;

4. Maximum bulk pressure method;

5. Methods analyzing shape of the hanging liquid drop or gas bubble;

6. Dynamic methods;

7. Hanging drop method;

O método mais frequentemente usado para determinar a tensão superficial sob condições extremas de pressão é a técnica de hanging drop method (pendant drop technique). Esta técnica será a usada para a construção do aparato experimental proposto no presente trabalho, uma vez que ela não depende de equipamentos que precisam ser calibrados, como transdutores mecânicos ou eletrônicos ou mesmo sensores de força.

Essa técnica oferece uma solução simples e elegante para determinar a tensão superficial e interfacial - um parâmetro central em muitos sistemas coloidais, incluindo emulsões, espumas e fenômenos de molhabilidade. A técnica envolve a aquisição de uma silhueta de uma gota de fluido axissimétrica e ajuste iterativo da equação de Young-Laplace que equilibra a deformação gravitacional da gota com a tensão interfacial restauradora.

Desde o advento de câmeras digitais e computadores de mesa de alta qualidade, esse processo foi automatizado com alta velocidade e precisão. No entanto, apesar de sua 
simplicidade atrativa, ainda existem complicações e limitações, associadas ao equilíbrio entre a tensão interfacial e as forças gravitacionais que acompanham o experimento e o volume da gota. A importância das forças gravitacionais em comparação com as forças de tensão superficial é medida pelo número de Bond ou de Eötvös. (RABÓCZKAY, 2016).

A tensão interfacial é uma das propriedades termodinâmicas fundamentais que controlam o comportamento dinâmico de fluxos multifásicos e um dos fatores mais importantes para simulações de alta precisão. Dessa forma, neste estudo, foram modelados e discutidos os processos envolvidos em ir de uma imagem experimental capturada a um valor de tensão interfacial ajustado, realçando características pertinentes e limitações ao longo do caminho.

\subsection{Materiais}

Os equipamentos utilizados, especificações técnicas e informações relevantes podem ser resumidas como:

1. Um cilindro de aço inox com capacidade limite de aproximadamente $250 \mathrm{ml}$;

2. Um compressor com vazão de $10000 \mathrm{~cm}^{2} / \mathrm{min}$ e potência de $50 \mathrm{~W}$;

3. Um manômetro analógico com um limite de medida aproximado de $3 \mathrm{Mpa}$;

4. Um termômetro analógico com um limite de medida aproximado de $770 \mathrm{~K}\left(500{ }^{\circ} \mathrm{C}\right)$;

5. Uma câmera digital embutida em um celular, com capacidade de 12 megapixels;

6. Um notebook com memória gráfica dedicada de 2 gigabytes, usado no tratamento das imagens.

Tendo em vista o contexto socioeconômico do estudo, todos esses equipamentos foram escolhidos por estarem disponíveis nos laboratórios da Universidade Federal Fluminense, então serão reutilizados de forma a não gerar custos atrelados. É um fato importante pois a crise causada pela pandemia dificultou bastante o acesso a recursos, ainda mais no campo de pesquisas.

\subsection{Proposta de Aparato}

Uma ilustração das peças e uma modelagem 3D do aparato proposto para a realização do experimento foram feitas com auxílio do software de modelagem SolidWorks $₫$ e estão disponíveis nas Figuras 1 e 2, respectivamente.

A construção é feita partindo da cruzeta $n^{\circ} 1$. No seu lado esquerdo será encaixado um visor, que permitirá enxergar a gota caindo. Alinhada ao visor, será posicionada a câmera. Os lados inferior e direito serão vedados, enquanto o lado superior será ligado à próxima cruzeta. Chegando à cruzeta $\mathrm{n}^{\circ} 2$, em seu lado esquerdo existirá uma pequena tubulação, para que seja posicionado o manômetro e o termômetro. O lado superior é associado à tubulação que liga ao cilindro e o lado direito possui outra tubulação que faz ponte com a cruzeta seguinte. A gota cairá através de um tubo que parte do lado superior da cruzeta $n^{\circ} 2$ e se estende até a linha de centro horizontal do visor.

Conectando a cruzeta $n^{\circ} 3$, em seus lados inferior e direito, existirá um conjunto tubulação com válvula, cada um com uma função bem definida, que serão tratadas no procedimento. A parte superior dela será conectada com a tubulação que seguirá até o lado direito da peça chamada de "Tê". A parte superior do tê terá um conjunto de tubulação com válvula e sua parte inferior será ligada ao cilindro, fechando o "circuito". A ligação e correção dos diâmetros entre visor, tubulações e vedações com relação às cruzetas e o tê é feita através de peças denominadas de bujões. O material do cilindro é composto de aço inoxidável, todas as tubulações são feitas de cobre e as cruzetas, tê e bujões são feitos de 
aço galvanizado para que seja possível lidar com a alta pressão e temperatura durante o experimento.

É importante destacar que o propósito é o projeto e a construção de um aparato experimental de construção simples, por meio de peças consideradas padrão na área de construção civil, minimizando os gastos com peças personalizadas ou muito sofisticadas e que, ainda assim, permitam que o experimento seja executado sem um déficit de qualidade grande com relação aos produtos e equipamentos mais rebuscados.

Figura 1 - llustração do aparato

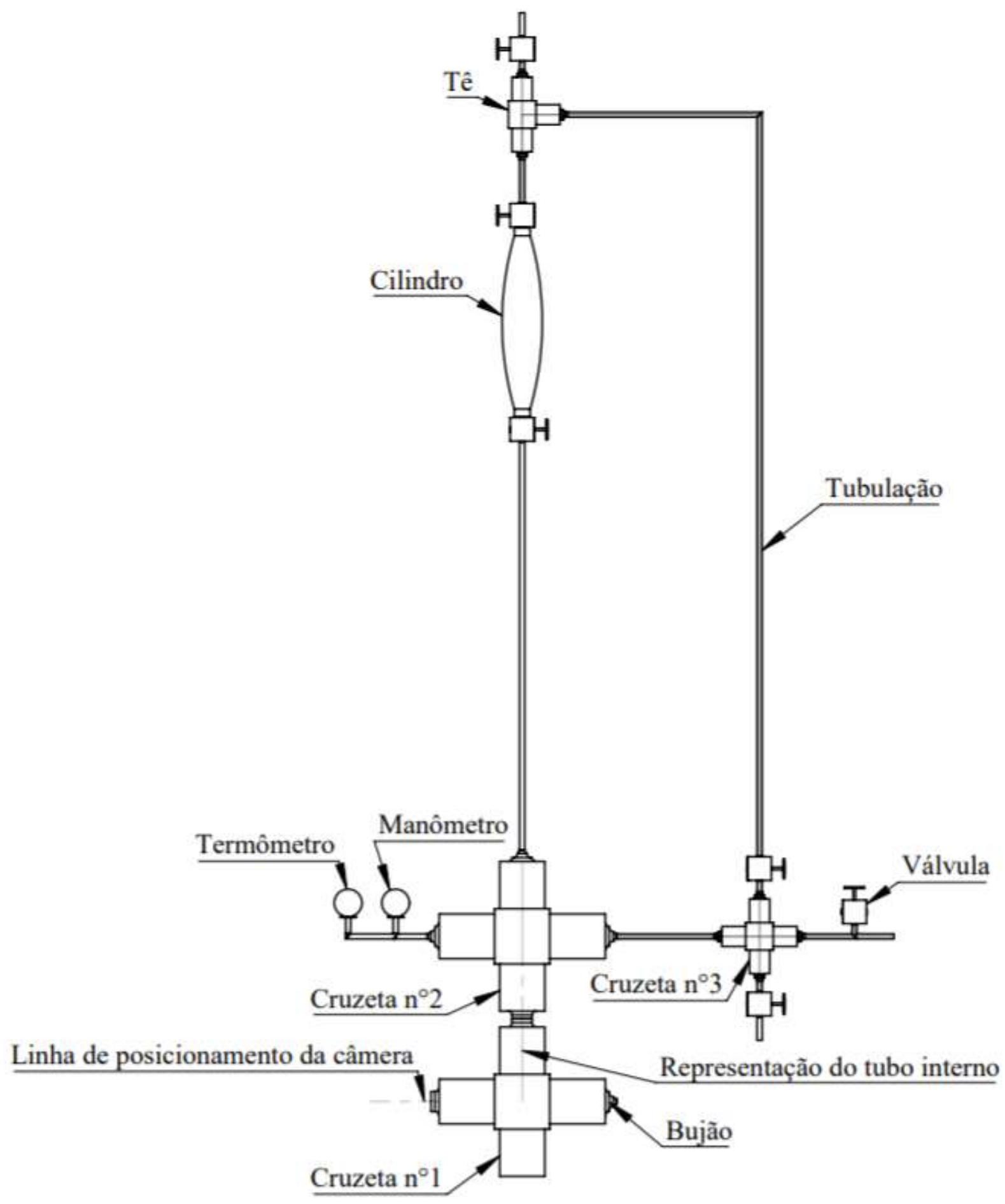

Fonte: Autores 


\section{COBENGE

Figura 2 - Modelagem 3D do aparato

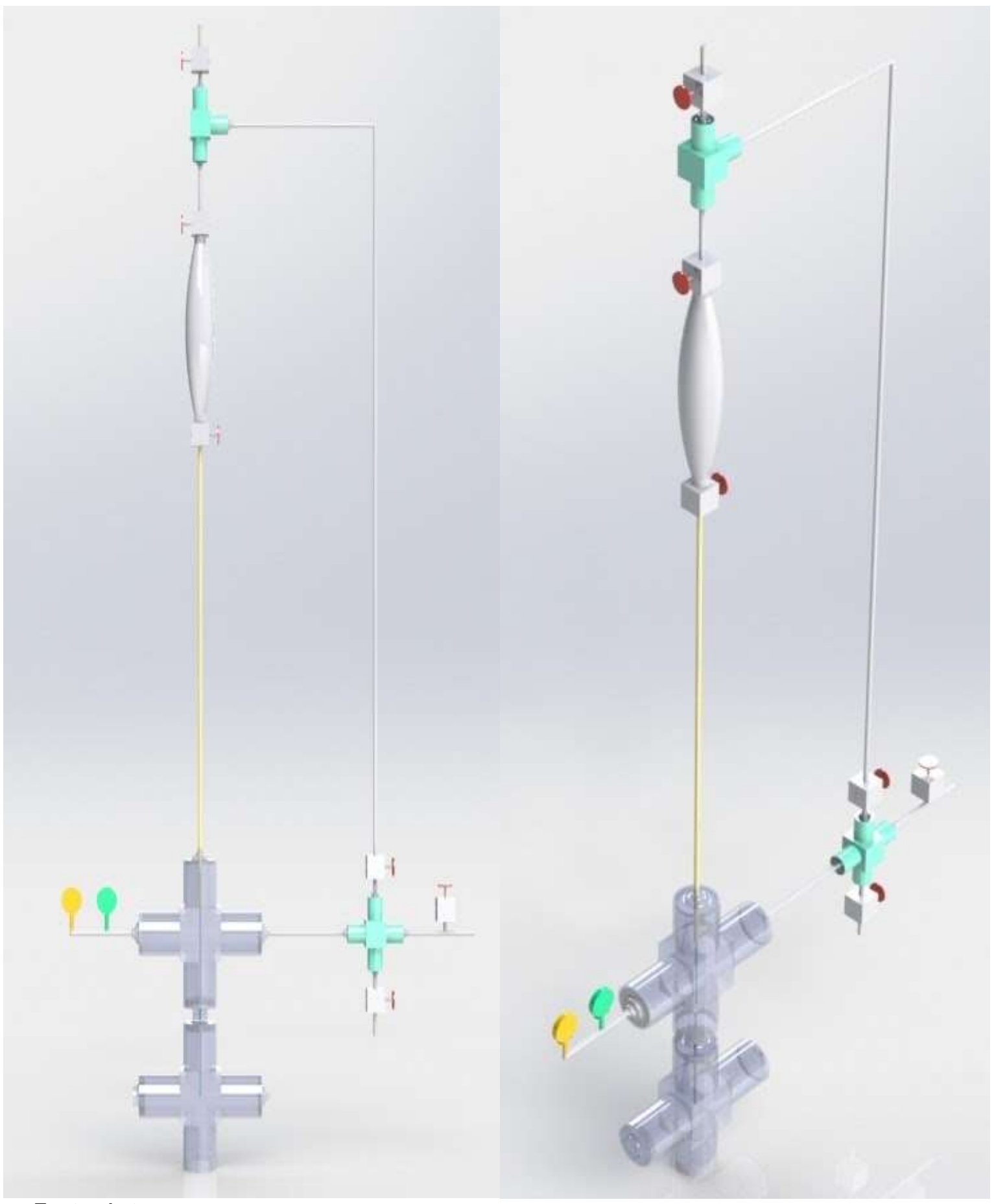

Fonte: Autores

\subsection{Resultados e discussão}

O presente artigo apresentou a descrição de um novo aparato experimental adequado para a medição precisa de tensão superficial e propriedades termodinâmicas aparentes em um sistema onde as características são voláteis e mudam dramaticamente com a mudança de pressão e temperatura. 
Foi demonstrado que o aparelho funciona bem para a análise do sistema e que as medições resultantes se comparam bem aos dados da proposta. Além disso, também foi demonstrado que, a partir desse experimento, é plausível a aplicação em diversas áreas, não só para a solução de problemas existentes, mas também para a evolução nessas áreas. Assim, como tanto a comparação com os dados da teoria quanto os resultados dos cálculos teóricos são extremamente bons, conclui-se que a técnica experimental está validada.

Como salientado, a técnica de tensiometria usada para utilização no aparato experimental proposto possui uma característica atraente porque se associa às relações entre as forças gravitacionais e a tensão interfacial. A gravidade é responsável pela deformação usada para quantificar a tensão interfacial, e a aceleração gravitacional é praticamente constante (com variações mínimas e facilmente quantificadas com a localização).

Desta forma, pretende-se que o equipamento projetado seja utilizado em combinações de abordagens experimentais e teóricas para diferentes situações na indústria de petróleo, incluindo aquelas que são relevantes para a recuperação aprimorada de petróleo e para o sequestro de $\mathrm{CO} 2$ e sua armazenagem em reservatórios de petróleo já exauridos, que estão fora de produção.

\section{DISCUSSÕES A RESPEITO DO PROJETO E SOBRE SUAS CONTRIBUIÇÕES PARA ATIVIDADES FUTURAS}

Os efeitos da pressão sobre as propriedades físico-químicas continuam a ser objeto relevante de uma grande quantidade de pesquisas, sendo a técnica de hanging drop method uma abordagem interessante para tal estudo.

Sua aplicabilidade se mostrou relevante não apenas nas ciências físicas, mas também na ciência dos alimentos, indústrias químicas, bioquímicas, de petróleo e metalúrgica. Há razões práticas e fundamentais para isso, visto que muitas reações químicas e processos são realizadas em alta pressão e, também, as medições sob pressão são simples e maneiras convenientes de estudar as propriedades moleculares do fluido.

Ainda, aplicando uma pequena oscilação de volume à gota, informações sobre a elasticidade e a resposta da camada superficial podem ser obtidas e isso tem potencial significativo no estudo de polímeros, proteínas e outras biomoléculas em interfaces, embora seja necessário tomar cuidado para levar em conta adequadamente os efeitos do fluxo de fluido e cisalhamento interfacial em tais experimentos.

Com a ciência se tornando significativamente mais acessível nos últimos anos, e com o advento da indústria 4.0, é provável que os sistemas dinâmicos de medição da tensão superficial se tornem mais relevantes e acessíveis, tornando-se mais presentes no âmbito de foco das pesquisas.

O método hanging drop é uma técnica poderosa que pode ser usada para determinar com precisão a tensão interfacial. Neste trabalho, fornecida uma discussão abrangente do embasamento teórico da técnica, destacando também suas limitações. Ainda, como destaque, mostra-se em que aspectos essa é facilitadora, ao trazer a aquisição precisa de dados de tensão superficial e interfacial, fornecendo oportunidades para desenvolver e expandir a própria técnica. Com esse artigo, pretende-se estimular o interesse de pesquisas adicionais em tensiometria de gota pendente, além de fornecer uma ferramenta para outros cientistas interessados.

\section{SARS-COVID-2}

6 O DESAFIO DA EXPERIMENTAÇÃO NO CONTEXTO DA PANDEMIA DO 
Historicamente, de forma geral, pode-se dizer que muitas atividades foram realizadas antes mesmo que fossem entendidos os conceitos teóricos envolvidos. Olhando para o uso do petróleo por exemplo: este é datado desde a época da antiga babilônia na aplicação de assentamento de tijolos e calafetação de embarcações, assim como os povos do Egito utilizaram na pavimentação de estradas e na construção de pirâmides, tudo isto antes mesmo da composição química do óleo ser de fato conhecida (THOMAS, 2012).

O aprendizado prático é intrínseco ao processo evolutivo da humanidade e é de suma importância para a execução de qualquer atividade, ainda mais no âmbito da engenharia. A experimentação ocupa lugar de destaque na vida acadêmica, ao favorecer a construção de vínculos entre teoria e prática e a construção de discurso científico, ao mesmo tempo em que estimula a socialização do conhecimento entre os estudantes que compõem uma mesma equipe. Assim, tendo em vista a relevância deste aspecto, os profissionais em formação são apresentados aos laboratórios desde o início da sua graduação, pois este é um ambiente propício à aquisição de conhecimentos e resolução de problemas desta natureza, através de experimentos.

Observando por exemplo o caso do curso de engenharia de petróleo da Universidade Federal Fluminense, para garantir que os alunos adquiram experiência neste campo, o currículo 51.01.003 oriundo da resolução N. ${ }^{\circ}$ 356/2014 do Conselho de Ensino e Pesquisa (CEP) prevê uma carga horária total de 3907 horas, sendo destas, 499 horas de cunho prático. É interessante observar que, se for considerado uma média de 2 horas por aula, os alunos frequentam o laboratório 250 vezes durante sua formação, ressaltando mais uma vez o quão essencial é este tipo de aprendizado.

Entretanto, com o surgimento da pandemia do sars-covid-2, veio a suspensão imediata de todas as atividades presenciais, o que inviabilizou a presença dos estudantes nos ambientes laboratoriais. Com uma estruturação bem pensada, a qualidade do ensino teórico é possível de ser mantida através do método de educação a distância, porém, quando se trata do campo prático, a "compensação" se torna muito mais complexa.

Nesse sentido, uma vez que os estudantes não possuem a maior parte dos recursos materiais necessários para a realização de diversas práticas, este estudo foi pensado como um caminho para desenvolver as competências experimentais, buscando se aproximar das atividades presenciais na medida do possível, tendo em vista o contexto atual. Fazendose, portanto, utilização dos recursos disponíveis, como por exemplo as literaturas de referência aliadas a um software de modelagem, que ainda assim favorecessem a investigação, a hipotetização e o desenvolvimento de habilidades de argumentação e criticidade.

\section{CONSIDERAÇÕES FINAIS}

A pandemia do novo coronavírus trouxe uma realidade e desafios nunca antes presenciados. Isso levanta dentro da realidade educacional novas dificuldades, restrições práticas, orçamentais e estruturais. Depois de fechar salas de aula e laboratórios por mais de um ano, as universidades e seus professores, acadêmicos e funcionários, precisam mostrar a sua capacidade de se reinventar. Além disso, esse novo cenário circunstancial também passou pela produção científica.

Fazer ciência é um processo complexo que envolve não só pessoas, mas também estrutura física, tempo dedicado, testes, erros e sucesso. Normalmente, também é necessário começar de novo, encontrar maneiras de ver os resultados de uma perspectiva diferente, e reaprender a ler o que foi descoberto. Por todas essas razões, a pesquisa não 
é feita da noite para o dia e conduzi-la nas circunstâncias atuais provou ser ainda mais desafiador, de modo que se tornou evidência de um processo resiliente.

\section{REFERÊNCIAS BIBLIOGRÁFICAS}

\section{CAMPOS, P. R. B. DE. O ENSINO REFLEXIVO EM EXPERIMENTOS DE LABORATORIO DIDATICO NA ENGENHARIA, 2012.}

JACOBSEN, Priscila. O desafio de fazer ciência em casa. Biblioteca Central UFRGS BLOG. Rio Grande do Sul. $2020 . \quad$ Disponível em:<https://www.ufrgs.br/blogdabc/desafiodefazercienciaemcasa/\#: :text="Os\%20pesquis adores $\% 20$ focam $\% 20$ em $\% 20$ atividades, apresentar\%20os $\% 20$ resultados $\% 20$ do\%20estud o. Acesso em: 01 maio 2021.

KODERA, M. et al. Interfacial tension between decane saturated with methane and water from $283.2 \mathrm{~K}$ to $298.2 \mathrm{~K}$ under pressures upto $10 \mathrm{MPa}$. Journal of Industrial and Engineering Chemistry, v. 81, p. 360-366, 2020.

RABÓCZKAY, T. Fisico-quimica De Interfases. EDUSP, 2016.

SHAW, D. Introduction to Colloid and Surface Chemistry. Elsevier Science, 1992.

THOMAS, J. E. Fundamentos de Engenharia de Petróleo, 2012.

TIAB, D.; DONALDSON, E. C. Petrophysics - Theory and Practice of Measuring Reservoir Rock and Fluid Transport Properties, 2015.

\section{THE CHALLENGE OF GRADUATION IN PETROLEUM ENGINEERING IN THE CONTEXT OF THE SARS-COV-2 PANDEMIC: A PROPOSAL FOR PERFORMING EXPERIMENTS ON SURFACE TENSION}

Abstract: Considering the difficulties imposed by the sars-covid-2 pandemic, this work seeks to facilitate the feasibility of an experiment relevant to the oil and gas segment, through the proposal of a simple construction apparatus. The chosen study was the measurement of surface and interfacial tension, which is important in phenomena that occur at high temperatures and pressures, using the drop weight method. Surface and interfacial tension is a quantity with significant potential to provide relevant information to the solution of some problems present in the oil industry. In the development, the relevance, challenges, and structuring of the experiment are presented. Finally, a reflection is made on the significance of this study from the perspective of the parties involved.

Keywords: Laboratory. Surface and interfacial tension. Oil industry. Experiment. Sarscovid-2 pandemic. 